\title{
Computer Vision Technology using Gesture Recognition
}

\author{
Mohaney Manasi Gajanan \\ M.Tech Student \\ All Saints College of Technology \\ Bhopal (M.P), India
}

\author{
Sonal Chaudhary \\ Assistant Professor \\ All Saints College of Technology \\ Bhopal (M.P), India
}

\begin{abstract}
In this paper, an individual human PC interface framework utilizing face and eye movements is presented. Generally, human PC interface utilizes mouse, console as an information gadget. This paper presents hands free interface amongst PC and human. The paper introduces an original plan to control PC mouse cursor development with human face and eyes. It controls mouse-moving via naturally influencing the position where visual perception concentrates on, and recreates mouseclick by staying cursor on single position. The accuracy of eye tracking was found to be approximately one degree of visual angle. It is expected that the availability of this system will facilitate the development of eye- tracking and face-tracking applications and the eventual integration of face and eye tracking into the next generation of everyday human computer interfaces.
\end{abstract}

\section{Keywords}

Camera, Viola Jones, Mouse tracking.

\section{INTRODUCTION}

It is a fact that the computer has contributed and continues to contribute to the evolution of society as a whole. Using a computer can make life easier for people in several ways, from day to day problem solving to people interaction via communication channels over the internet. "Nowadays it is known that Communication and Information Technologies are increasingly becoming important instruments in our culture, and its use is a real mechanism of inclusion and interaction around the world. Communication and Information Technologies (CIT) are more conspicuous when used for the development of applications aimed at the social inclusion of handicapped people in our contemporary society. However the main limitation of this device is the lack of usability for the people with disabilities including limb paralysis and those who lose their limbs in any accidents or poor development of body due to congenital defects. Such users face problems and inability to hold or moving the mouse as how normal users perform it. The eye tracking systems give them enhanced usability by tracking the eye movements to capture the details of relative position of the eye of the disabled users and use the information to move the mouse on the screen and help them use computers easily in spite of their disabilities. Thus, thinking that a device capable of identifying deliberate movements of the eye area (pupils, eyelids and eyebrows), can provide a new means of interaction that could replace or complement more standard interfaces. Face recognition system is one of the applications of a computer for verifying and identification of a person. This field is related to biometrics application which includes others like fingerprint, eye iris recognition systems. Face Recognition systems are based on an very old era of $2 \mathrm{D}$ algorithms, taking us back to the early 1960s. The first face recognition methods used the geometry of key points. Hand Tracking is technique used to move and control mouse cursor without using an external mouse device. Our paper proposes a basic model of integrating Face Recognition method with Hand Tracking where the authenticated user alone can access their system without using mouse thereby providing access privilege to the user. Hand Tracking is been carried out with either Edge/Shape Motions acknowledgment framework progressively getting to be noticeably huge piece of humanPC cooperation. Motions can begin from any substantial movement or state yet usually start from the face as well as hand.

Human eye structure: A human eye is an organ that senses light. An image of eye anatomy is shown in Figures. Several important parts of human eye related to eye tracking are described here. The cornea is a transparent coat in front of eyeball. The iris is the muscle that controls the size of pupil, which is like the aperture in a camera to let light goes inside. The iris has color and is different from person to person, thus can be used in biometrics. The sclera is the tough outer surface of the eyeball and appears white in the eye image. The limbus is the boundary between the sclera and the iris. An image of eye captured by digital camera is shown in Figure below.

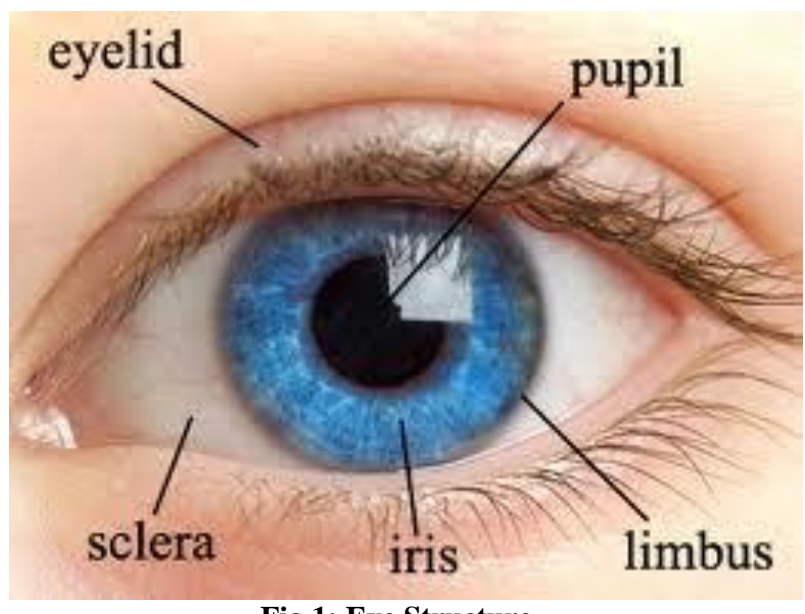

Fig 1: Eye Structure

\section{LITERATURE SURVEY}

H. Mosquera, Humberto Loaiza, Sandra Nope, Andrés Restrepo proposed a framework which was created utilizing facial signals caught by the web camera performs control over the working framework, permitting peruse the Web without having physical contact with the $\mathrm{PC}$ or require extra equipment. The framework utilizes make a beeline for move the mouse and signal to close an eye to actuate the snap. The semi-straightforward graphical interface made permits common and instinctive route, open and move between the route tabs, embed content, seek, vertical parchment, zoom control, turn back and exit. 
Parth B. Pancha, Vimal H. Nayak proposed a research in which a close by motion acknowledgment expects to plan and improvement of such frameworks that can distinguish unequivocal human motions as info and can process these motion portrayals through mapping of orders as yield for gadget control. The touch screen LCD can be used to show different diverse messages and it can be change as per change in accelerometer position or distinctive edges of accelerometer.

Danchi Huang, and Lijuan Li proposed a technique in which protest tracking can be done accurately, in spite of the fact that it is impeded by the comparable skin-shading objects. In this, utilizing the twice-coordinating to coordinate the question, and refresh the format, with the goal that the protest can discover effectively when the presence of the protest is changed. When the protest is lost, the calculation can discount it rapidly and keep following. Improvement of this calculation is expected to better match the protest with different highlights.

Prajakta Tangade, Shital Musale, Gauri Pasalkar, Miss. Umale M.D., Miss. Awate S.S. in this user's eye gaze is used to control the computer cursor. The control keys which are displayed on screen, a person can synthesize speech, control the environment type, operating a telephone, executing computer software, handling a computer mouse, and access the internet and e-mail by looking at control keys. The eyegaze system is a direct-select vision-controlled communication and control system. The only requirement that is used to operate the Eye gaze is at least one eye with good vision and to keep head still. The system provides fair results and uses external device for voice command and also external noise from surrounding is reduced.

Arslan Qamar Malik, and Jehanzeb Ahmad used Electrooculography (EOG) technique in which eye retina is used for moving the mouse cursor. The movement of mouse cursor is controlled by sensing the eye signal sensed by EOG. The signals captured using electrode sensors, before being transferred to PC for software interfacing, are first amplified, then noise is removed and then digitized. The EOG is the technique used for retina tracking. For recording the eye movements the EOG electrodes are placed on user's forehead around the eyes. Electrodes are used for detecting EOG which is a very small electrical potential. The electrodes are placed in left, right, up and down so whenever the retina moves from center to towards the electrodes, these electrodes results in positive side that in turns move the mouse cursor in respective direction. This method uses external electrical circuit for tracking the retina movement. The output results i.e. the movement of the cursor is limited to left, right, up and down. The system cannot be implemented for cursor movement top corners and bottom corners.

Dhairya Vyas and Hardik N. Mewada in this the software is developed for face and retina detection. To get the desired result this software is simulated in Matlab and OpenCV. The face and retina tracking is done by using Viola-Jones object detection algorithm that later used for detecting the human face. Thousands of human faces are passed through the dataset for training this algorithm. After training this algorithm it can identify whether the given image contains human face or not. By using the in build function of OpenCV this algorithm is easily implemented. This algorithm provides the successful result in real-time simulation.

Shrunkhala Satish Wankhede, Ms. S. A. Chhabria, Dr. R. V. Dharaskar this paper describes the various ways in which the tracking of eye retina is done and describes how the blink detection work. The various face detection technique is used, which can be done in two ways,

Feature-based method is used detect the facial features like nose, eyes, mouth, etc. This detection is done to verify that the given image contains human face or not.

Image-based method technique is used for template matching to detect the human face. The various methods of face detection show desire output and provide the good results.

\section{PROPOSED ALGORITHM}

The gesture recognition based on computer vision technology has been studied and implemented using viola Jones algorithm. The input video is taken from camera. The input image is converted into RGB TO GRAY format. The gray plane image consists of maximum information to extract face and eye feature. The algorithm continuously tracks the video object which is chosen by the user. Those face or eyes are continuously tracked. If the cursor is still for at least 1 second then the key press event is processed. Detecting the key press virtual mouse driver is initiated.

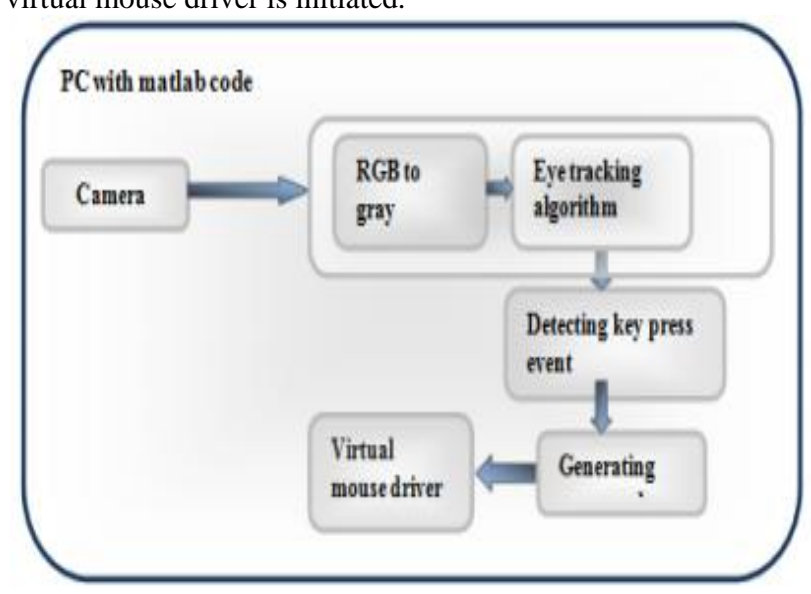

Fig 2: Block diagram of Gesture Recognition

\subsection{Viola-Jones Face Detection}

The Viola-Jones object detection framework is the first object detection framework to provide competitive object detection rates in real-time and later used for face detection. This framework is proposed in 2001 by Paul Viola and Michael Jones. Although it can be trained to detect a variety of object classes like human face, animal face, non-human face. It was motivated primarily by the problem of face detection. This algorithm can easily implemented in OpenCV by using in build function cvHaarDetectObjects(). The function cvHaarDetectObjects() is called as HaarCascade Classifier. This function is used for face detection as well as it is used for object detection. It is the combination of Haar Feature and Cascade Classification of Viola-Jones. The Haar Feature is the detection technique is based on the idea of the wavelet template that defines the shape of an object in terms of a subset of the wavelet coefficients of the image. In Matlab the system object vision.CascadeObjectDetector uses the ViolaJones algorithm to detect the faces, noses, eyes, mouth, or upper body. 


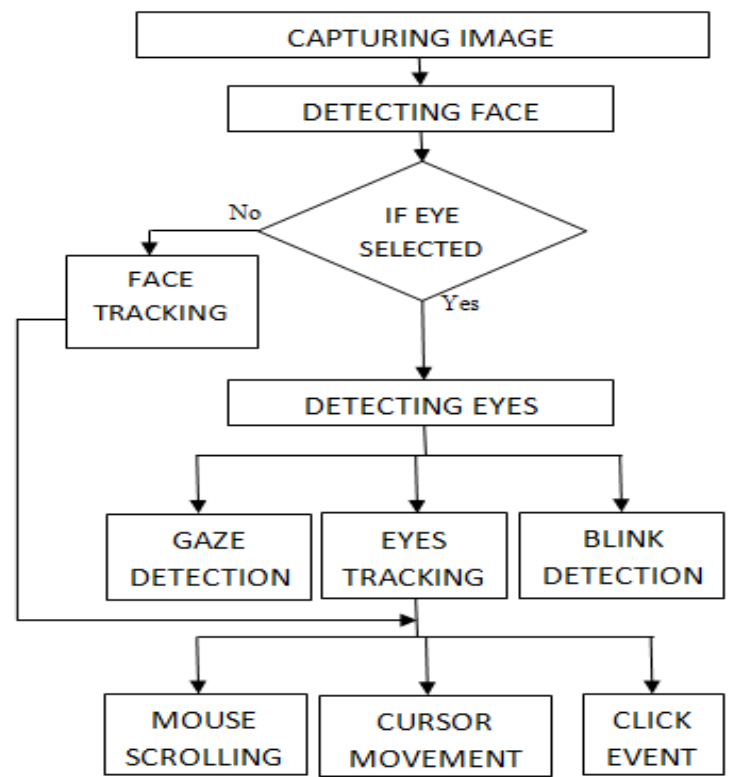

Fig 3: Flow Chart for Gesture Recognition

\section{CLASSIFICATION OF DIFFERENT GESTURE DETECTION TECHNIQUE}

There are various technique for detection of face and eye tracking and EOG systems for mouse control. Some of them are equipped with sophisticatedly designed systems which are discussed below.

\subsection{Face and Eye Movement tracking system}

By using the movement of the user's face, the movement of the mouse can be controlled, allowing face movement to be used as an ordinary computer mouse. But face tracking technique faces the problem that some disabled person cannot even move their face comfortably, and if the camera is not capturing the user's face then the system becomes inaccurate. In eye tracking a camera tracks the features of the eye and then mouse controlling is done accordingly.

\subsection{Hand Detection technique}

It is difficult to build a hand gesture-based interaction system. There are several ideas that can be used in appearance, shape, color, depth, and context. In problems like face detection, the appearance is a very good indicator. In image, hand is considered as the major portion. As the hand is the major part it would be easy to segment it by using the segmentation techniques proposed by Albiol et all. This method of segmentation is more related to human perception a sour eyes could easily recognize these kin tone from its background. This classical method sets upper and lower bound values for segmenting the skin pixels using which the hand was segmented. Removal of noise from the segmented image is absolutely necessary because it classifies skin as noisy objects. Before performing the recognition process the images are resized to a fixed resolution.

\subsection{Electro-oculography (EOG)}

In this technology electrodes are placed on user's forehead around the eyes to record eye movements. Electrodes are used to detect EOG which is a very small electrical potential. This setup used by majority of people caused severe cerebral palsy or been born with a congenital brain disorder or suffered traumatic brain injury, for example from automobile or drowning accidents. This technique is adapted because it is inexpensive and accurate. The anatomy of the eye is shown in the Fig.1. The light entering the pupil, is focused, inverted by the cornea and lens and projected onto the center of the eyes with the region of highest visual acuity. The seven layers of alternating cells and processes convert a light signal into a neural signal. The rods and cones are the actual photo receptors, but the cells that transmit to the brain are the ganglion cells.

\section{RESULT}

The interaction of human with computer can be configured by making GUI. The camera is interface with computer which detects the face or eye by the use of algorithm. The user has option to select the input parameter eye or face respectively. Fig.4 shows the GUI where the input is captured and mouse movement is tracked based on user's eye or face position.

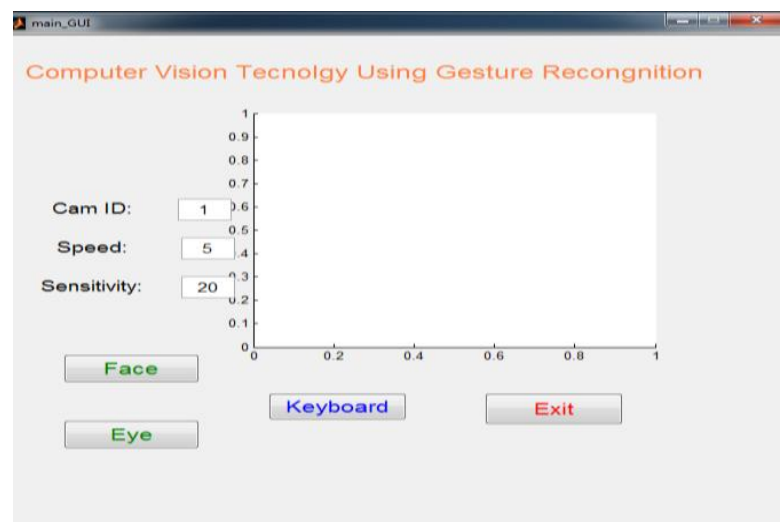

Fig.4 Gesture Based Human computer Interaction

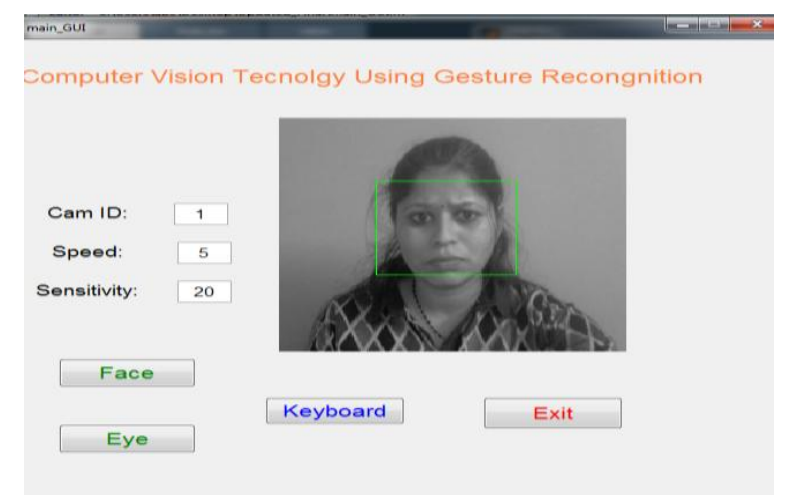

Fig.5 Movement of cursor according to face position

The face detection is shown in Fig.5 where the cursor is moved according to the face position. The eye detection is shown in Fig. 6 where the cursor is moved according to the eye position. 


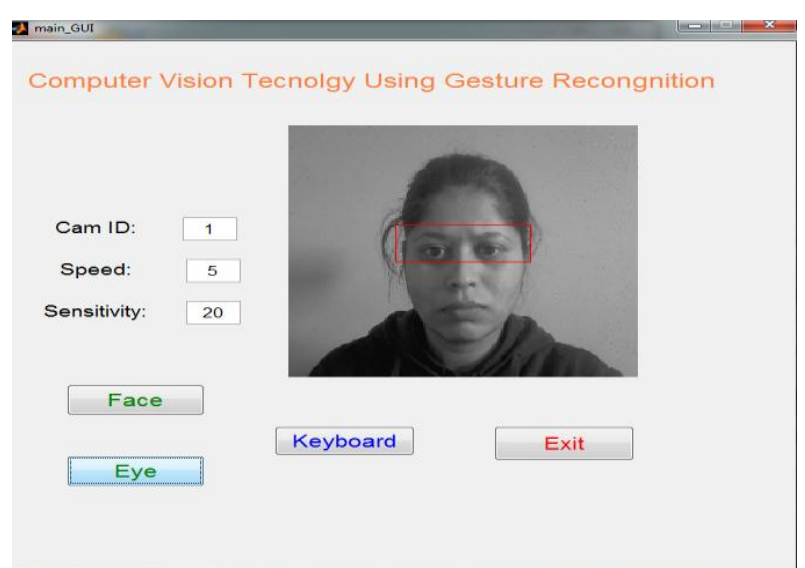

Fig.6 Movement of cursor according to eye position

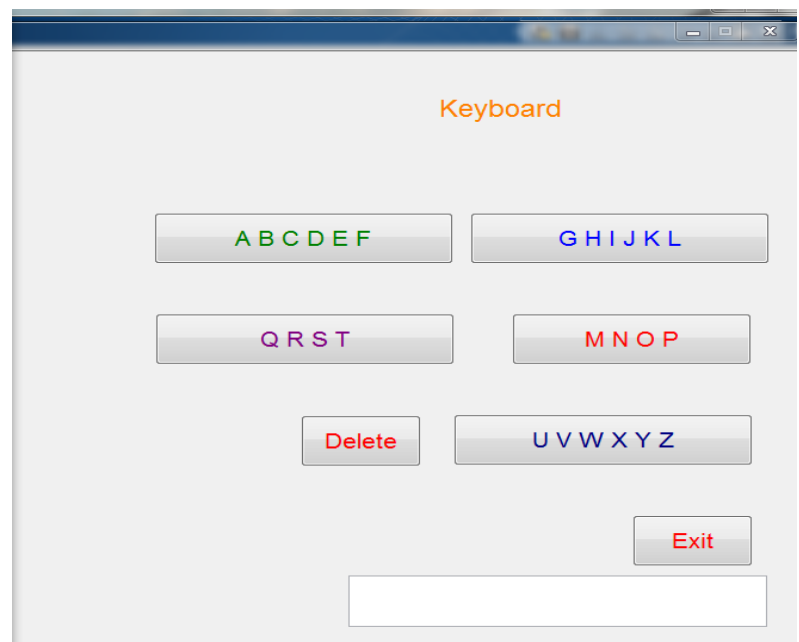

Fig.7 Occurrence of Click event for keyboard button

Fig.7 shows the click event occurred when the cursor was still for at least $1 \mathrm{sec}$ on keyboard button and the keyboard gets open. Table 1 shows the event occurred according to the face and eye movement.

Table 1. Mouse Click Event occurred by face and eye movement

\begin{tabular}{|c|c|}
\hline $\begin{array}{c}\text { Face and Eye } \\
\text { Movement }\end{array}$ & $\begin{array}{c}\text { Cursor Movement and event } \\
\text { occurred }\end{array}$ \\
\hline Left Movement & Cursor move towards Left \\
\hline Right Movement & Cursor move towards Right \\
\hline Up Movement & Cursor move Upwards \\
\hline Down Movement & Cursor move Downwards \\
\hline $\begin{array}{c}\text { Still for at least } \\
\text { 1sec on any files, } \\
\text { folder, links or } \\
\text { application }\end{array}$ & $\begin{array}{c}\text { Click event occurs on that file, folder, } \\
\text { link or application }\end{array}$ \\
\hline
\end{tabular}

\section{CONCLUSION}

Camera based face detection and tracking makes it easy for human computer interaction. This makes easy for mouse control. The non intensive technique makes it comfortable for user. The system is implemented for control parameters. The gesture can be detected and used as the future scope for controlling of PC or smart TV or any other controlled parameters and it can also be merged with various applications to perform mouse click event. The system can be controlled by using Face or Eyes movement

\section{REFERENCES}

[1] H. Mosquera, Humberto Loaiza, Sandra Nope, Andres Restrepo Universidad del Valle, "Identifying facial gesture to emulate a mouse control application in a web browser", Signal Processing, Images and Artificial Vision (STSIVA), 2016 XXI.

[2] Parth B. Pancha, Vimal H. Nayak, "A Hand Gesture Based Transceiver System for Multiple Application" IEEE Sponsored $2^{\text {nd }}$ international Conference on Electronics and Communication System (ICECS 2015).

[3] Danchi Huang, and Lijuan Li, "Face Tracking Algorithm Based on Improved Camshift and Surf Algorithm",Journal of Computational Information Systems 11: 3 (2015) 893 - 901.

[4] Prajakta Tangade, Shital Musale, Gauri Pasalkar, Miss. Umale M.D., Miss. Awate S.S., "A Review Paper on Mouse Pointer Movement Using Eye Tracking System and Voice Recognition." International Journal of Emerging Engineering Research and Technology Volume 2, Issue 8, 2014.

[5] Arslan Qamar Malik, and Jehanzeb Ahmad , "Retina Based Mouse Control (RBMC)" International Journal of Electronics, Circuits and Systems Volume 1 Number 4, 2004.

[6] Dhairya Vyas and Hardik N. Mewada, "Retina Based Mouse Control System" IJSRD - International Journal for Scientific Research \& Development Vol. 1, Issue 11, 2014.

[7] Shrunkhala Satish Wankhede, Ms. S. A. Chhabria, Dr. R. V. Dharaskar, "Controlling Mouse Cursor Using Eye Movement" International Journal of Application or Innovation in Engineering \& Management (IJAIEM), 2013.

[8] Shahzad Malik, "Real-time Hand Tracking and Finger Tracking for Interaction", CSC2503F Project Report, December 18, 2003.

[9] SaurabhS.Chakole; VivekR.Kapur, Y.A.Suryawanshi, “A RM Hardware Plaform for Vehicular Monitoring and Tracking" 2013 International Conference on Communication Systems and Network Technologies. 